Gut and Liver, Vol. 9, No. 3, May 2015, pp. 340-345

\title{
Additive Effect of Pronase on the Eradication Rate of First-Line Therapy for Helicobacter pylori Infection
}

Chang Seok Bang, Yeon Soo Kim, Sang Hyun Park, Jin Bong Kim, Gwang Ho Baik, Ki Tae Suk, Jai Hoon Yoon, and Dong Joon Kim

Department of Internal Medicine, Hallym University College of Medicine, Chuncheon, Korea

See editorial on page 257.

Background/Aims: Helicobacter pylori colonizes on the apical surface of gastric surface mucosal cells and the surface mucous gel layer. Pronase is a premedication enzyme for endoscopy that can disrupt the gastric mucus layer. We evaluated the additive effects of pronase combined with standard triple therapy for $H$. pylori eradication. Methods: This prospective, single-blinded, randomized, controlled study was conducted between June and October 2012. A total of 116 patients with $H$. pylori infection were enrolled in the study ( $n=112$ patients, excluding four patients who failed to meet the inclusion criteria) and were assigned to receive either the standard triple therapy, which consists of a proton pump inhibitor with amoxicillin and clarithromycin twice a day for 7 days (PAC), or pronase (20,000 tyrosine units) combined with the standard triple therapy twice a day for 7 days (PACE). Results: In the intention-to-treat analysis, the eradication rates of PAC versus PACE were $76.4 \%$ versus $56.1 \%$ ( $p=0.029$ ). In the per-protocol analysis, the eradication rates were $87.5 \%$ versus $68.1 \%(p=0.027)$. There were no significant differences concerning adverse reactions between the two groups. Conclusions: According to the interim analysis of the trial, pronase does not have an additive effect on the eradication of $H$. pylori infection (ClinicalTrial.gov: NCT01645761). (Gut Liver 2015;9:340-345)

Key Words: Helicobacter pylori; Pronase

\section{INTRODUCTION}

Helicobacter pylori is a Gram-negative bacterium which colonizes the gastric epithelium. Subsequent modification of acid secretion and gastric architecture by immune response result in various diseases of upper gastrointestinal tract such as gastritis, peptic ulcer, gastric cancer, and extranodal marginal zone lymphoma of mucosa-associated lymphoid tissue. However, currently, there is no uniform and definite therapeutic regimen for the $H$. pylori eradication due to the antimicrobial resistance to standard triple therapy. Recent data showed that decreased eradication rate less than $80 \%$ in most countries that is unacceptable regarding infectious disease which could promote severe outcome. $^{1-3}$

Pronase is a kind of proteolytic enzyme isolated from Streptomyces griseus in $1962 .{ }^{4}$ Since its first use as a premedication for X-ray diagnosis of stomach in 1964, it has been applied to endoscopic premedication for enhanced visibility of gastric mucosa. $^{5,6}$ It can disrupt and make a reduction in the thickness of surface mucous gel layer (SMGL). ${ }^{7-9} H$. pylori colonizes on the apical surface of gastric surface mucous cells and the SMGL. ${ }^{10,11}$ In particular, H. pylori colonizes the SMGL preferentially, during antimicrobial treatment maintenance period. ${ }^{11}$ Potential hypothesis was whether the disruption of SMGL by pronase could enhance the eradication rate of $H$. pylori infection by making the organism inhospitable on the stomach. ${ }^{7}$ Based on this concept, additive effect of pronase combined with the standard triple therapy for the $H$. pylori eradication was evaluated.

\footnotetext{
Correspondence to: Jin Bong Kim

Department of Internal Medicine, Hallym University Chuncheon Sacred Heart Hospital, Hallym University College of Medicine, 77 Sakju-ro, Chuncheon 200-704, Korea

Tel: +82-33-240-5811, Fax: +82-33-241-8064, E-mail: kimjinbong@hallym.or.kr

Received on October 21, 2013. Revised on January 2, 2014. Accepted on January 22, 2014. Published online on June 18, 2014

pISSN 1976-2283 eISSN 2005-1212 http://dx.doi.org/10.5009/gnl13399

Chang Seok Bang and Yeon Soo Kim contributed equally to this work.

@ This is an Open Access article distributed under the terms of the Creative Commons Attribution Non-Commercial License (http://creativecommons.org/licenses/by-nc/3.0) which permits unrestricted non-commercial use, distribution, and reproduction in any medium, provided the original work is properly cited.
} 


\section{MATERIALS AND METHODS}

\section{Study design}

This was a prospective, single blind, single center, randomized controlled study. The eligible patients with $H$. pylori infection were randomly assigned to receive either the standard triple therapy, which consists of proton pump inhibitor (PPI) with amoxicillin $(1,000 \mathrm{mg})$ and clarithromycin $(500 \mathrm{mg})$ twice a day for 7 days (PAC) or the Endonase ${ }^{\circledR}$ (pronase 20,000 tyrosine units; Pharmbio Korea Co., Ltd., Chungju, Korea) twice a day combined with standard triple therapy for 7 days (PACE). The coadministrating agent $\mathrm{NaHCO}_{3}(1 \mathrm{~g})$ was prescribed in the PACE group. The administration method was taking all four drugs with $\mathrm{NaHCO}_{3}$ powder at the same time. The pretreatment $H$. pylori status was assessed by rapid urease test, ${ }^{13} \mathrm{C}$-urea breath test and histology. The posttreatment $H$. pylori status was assessed by ${ }^{13} \mathrm{C}$-urea breath test. $H$. pylori eradication was assessed at least 4 weeks after finishing the eradication medication. All the patients were educated by doctors who prescribed the medication and by research nurses; they were informed about the drug, administration time, possible adverse events and how to report the adverse reactions. The eradication rates of $H$. pylori infection, adverse reactions and compliance were investigated and compared with each other. The study protocol adhered to the ethical guidelines established by the 1975 Declaration of Helsinki and had received an approval by the Ministry of Food and Drug Safety and the Institutional Review Board for human research at Chuncheon Sacred Heart Hospital before the study was initiated (2011-74). This study was registered at ClinicalTrial.gov in July 2012 (Clinical trial registration number, NCT01645761). Informed consent to participate in the study was obtained from each patient.

\section{Outcome measures}

Primary endpoint was to compare the eradication rate of the 7-day standard PPI-based triple therapy plus pronase with that of the 7-day standard PPI-based triple therapy. Secondary endpoint was to investigate the difference in the number of participants with adverse events between patients receiving standard triple therapy plus pronase and patients receiving control treatment.

\section{Randomization}

A single independent staff prepared the randomization sequence, which was accomplished by using a block design and a block size of 4. Randomization of block was done by means of the random-number chart. This study was single blind trial due to the unique aroma and taste of Endonase ${ }^{\circledR}$ which challenged the successful blinding of the patients. Doctors did not know the result of the allocation; however, the patients were aware of the drugs they were prescribed and were asked not to give the information to the doctors about the medication.

\section{Study population}

This study was conducted at Chuncheon Sacred Heart Hospital, a tertiary center. Between June 2012 and October 2012, consecutive patients who were diagnosed with $H$. pylori infection were asked to participate in this study. Hemorrhage is the one of the adverse reactions of pronase. Thus, only those patients with peptic ulcer disease (PUD) of scar stage or nonulcer dyspepsia (NUD) and those who were treatment naive were enrolled. Patients with PUD of active or healing stage and those who took medications as PPI, Histamin-2 receptor blocker and antibiotics within 4 weeks, who underwent gastric surgery and less than 18 years of age, were all excluded from this study. Informed consent was taken from each patient by physicians.

\section{Assessment of the H. pylori infection}

The $H$. pylori infection status was assessed by one or more than one of the following methods: rapid urease test, ${ }^{13} \mathrm{C}$-urea breath test and histology. Two specimens from each of the gastric corpus and antrum were taken for rapid urease test (Pronto Dry; Gastrex Corp., Warsaw, Poland) or histological assessment using Giemsa staining during endoscopy. $\mathrm{A}{ }^{13} \mathrm{C}$-urea breath test (UBiT-IR 300; Otsuka Pharmaceutical Co., Ltd, Tokyo, Japan) with measurement of exhaled ${ }^{13} \mathrm{CO}_{2}$ before and 30 minutes after ingestion of ${ }^{13} \mathrm{C}$-marked urea $75 \mathrm{mg}$ were performed. An initial breath sample was obtained after at least an 8-hour fasting (overnight fasting). The ${ }^{13} \mathrm{C}$-urea breath test after the eradication of $H$. pylori was performed at least 4 weeks after the end of the eradication therapy. Delta over baseline $>4 \%$ was considered positive.

\section{Statistical analysis}

Sample size calculation was as follows: (1) The eradication rate of $H$. pylori infection of 7 days standard triple therapy was reported as 75\% in Korea. ${ }^{12}$ (2) The expected enhancement of the eradication rate of endonase combined with the triple therapy was assumed as 15\%. The number of patients required for the study with a two-tailed 5\% significance test and a power of 80\% with 10\% drop rate was 108 in each group. In the first protocol, there was no predetermined terms for interim analysis. However, due to the ethical issue from unexpected low eradication rate in the 7-day standard PPI-based triple therapy plus pronase, a protocol amendment was approved, establishing an interim analysis after inclusion of 50\% of the patients.

For the intention-to-treat (ITT) analysis, all patients who took the prescribed eradication medications and who checked the posttreatment $H$. pylori status were included and assessed. For the per-protocol (PP) analysis, only those patients who maintained and ended the prescribed eradication medications without violating the regulations (lost to follow-up visit or less than 85\% medication compliance) were included and assessed. The adherence was defined by taking more than $85 \%$ of the to- 
tal prescribed medications. The Student t-test and Fischer exact test were used to compare the continuous and categorical variables. The Mann-Whitney test was used if the variable did not show normal distribution in the continuous variables. A p-value $<0.05$ was considered to be statistically significant. Analysis was performed using the SPSS software version 18.0 (SPSS Inc., Chicago, IL, USA).

\section{RESULTS}

\section{Characteristics of patients}

Of the 116 eligible patients initially enrolled in this study, four patients were excluded due to their refusal to participate; as a result, a total of 112 patients ( 55 male and 56 female) participated. The characteristics of enrolled patients are summarized in Table 1 . They were randomly allocated (55 patients in PAC vs 57 patients in PACE). After finishing the eradication therapy, seven patients in PAC and 10 patients in PACE group were lost to follow-up. Finally, 95 patients (48 patients in PAC vs 47 patients in PACE) were included in the PP analysis. A study flow diagram is demonstrated in Fig. 1.

\section{The eradication rate}

A total of 112 patients were included in the ITT analysis and 95 patients in the PP analysis. For the PP analysis, 17 excluded patients were equally distributed between the PAC and PACE groups (12.7\% vs $17.5 \%, \mathrm{p}=0.60)$. Seven-day standard triple therapy (PAC) showed significantly higher eradication rate in both the ITT (76.4\% vs 56.1\%, p=0.029) and PP analysis (87.5\% vs $68.1 \%, p=0.027$ ) compared to pronase combined with standard triple therapy (PACE) (Table 2). In the subgroup analysis, there was no significant difference in the eradication rate between PUD and NUD both in the PAC and PACE group (Table 3).

Table 1. Characteristics of the Enrolled Population

\begin{tabular}{lccc}
\hline \multicolumn{1}{c}{ Variable } & $\begin{array}{c}\text { Standard triple } \\
\text { therapy } \\
(\mathrm{n}=55)\end{array}$ & $\begin{array}{c}\text { Pronase combined } \\
\text { with standard triple } \\
\text { therapy }(\mathrm{n}=57)\end{array}$ & $\begin{array}{c}\mathrm{p}- \\
\text { value }\end{array}$ \\
\hline Age, yr & $49.7 \pm 10.9$ & $48.5 \pm 12.4$ & 0.59 \\
Sex, male/female & $27 / 28$ & $31 / 26$ & 0.71 \\
Smoking & $8(14.5)$ & $6(10.5)$ & 0.58 \\
Alcohol & $24(43.6)$ & $23(40.4)$ & 0.85 \\
Peptic ulcer & $20(36.4)$ & $13(22.8)$ & 0.15 \\
Nonulcer dyspepsia & $35(63.6)$ & $44(77.2)$ & 0.15 \\
BMI & $24.8(23.1-27.4)$ & $23.2(21.45-25.3)$ & $0.01^{*}$ \\
\hline
\end{tabular}

Data are presented as mean \pm standard deiviation, median (interquartile range), or number (\%).

BMI, body mass index.

*Mann-Whitney U-test was used.
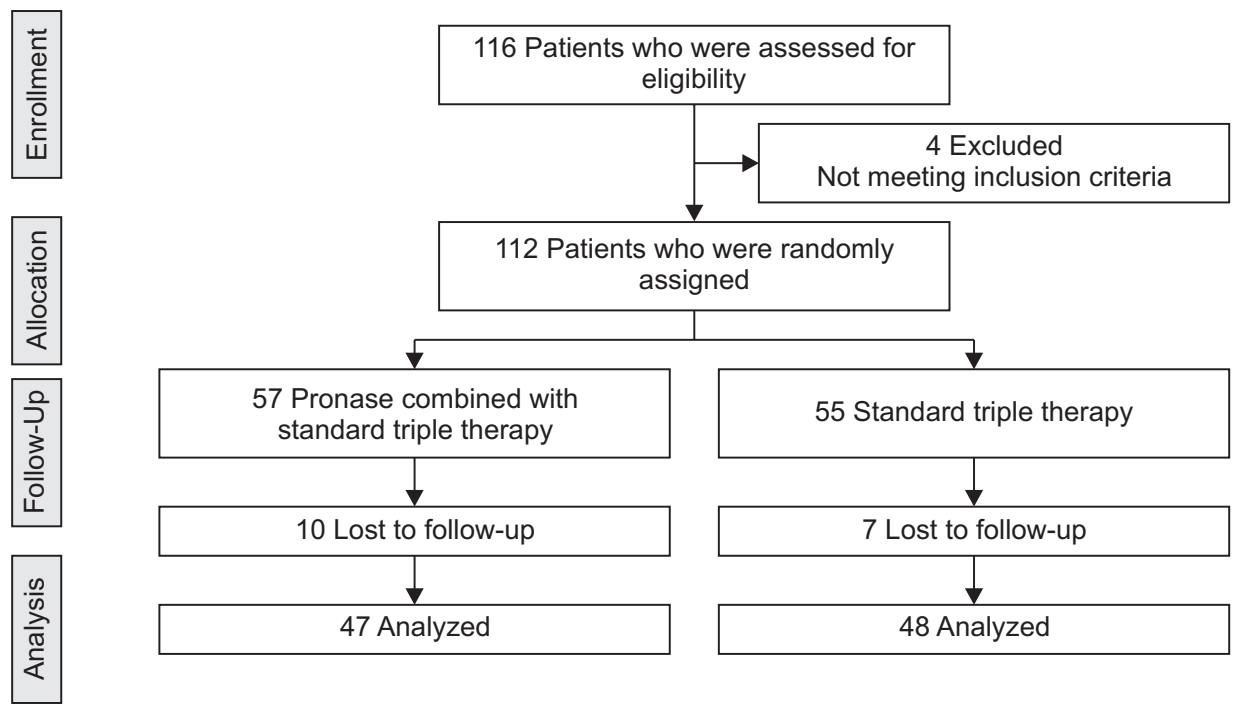

Fig. 1. Flow chart of the study design.

Table 2. Eradication Rates for Helicobacter pylori Infection

\begin{tabular}{|c|c|c|c|c|c|c|}
\hline \multirow[t]{2}{*}{ Variable } & \multicolumn{2}{|c|}{ Standard triple therapy } & \multicolumn{2}{|c|}{$\begin{array}{l}\text { Pronase combined with } \\
\text { standard triple therapy }\end{array}$} & \multirow{2}{*}{ OR $(95 \% \mathrm{CI})$} & \multirow[t]{2}{*}{ p-value } \\
\hline & Patients, $\mathrm{n}$ & Eradication rate, $\%$ & Patients, $\mathrm{n}$ & Eradication rate, $\%$ & & \\
\hline ITT & 55 & 76.4 & 57 & 56.1 & $2.52(1.12-5.69)$ & 0.029 \\
\hline PP & 48 & 87.5 & 47 & 68.1 & $3.28(1.15-9.40)$ & 0.027 \\
\hline
\end{tabular}

OR, odds ratio; CI, confidence interval; ITT, intention-to-treat; PP, per-protocol. 
Table 3. Eradication Rates between the Patients with Peptic Ulcer Disease and Those with Nonulcer Dyspepsia

\begin{tabular}{cccccccc}
\hline & \multicolumn{3}{c}{ Standard triple therapy } & & & \multicolumn{2}{c}{ Pronase combined with standard triple therapy } \\
\cline { 2 - 3 } & Patients, $\mathrm{n}$ & Eradication rate, $\%$ & $\mathrm{p}$-value & & Patients, $\mathrm{n}$ & Eradication rate, \% & $\mathrm{p}$-value \\
\hline PUD & 20 & 70 & 0.51 & & 13 & 61.5 & 0.54 \\
NUD & 35 & 80 & & 44 & 50 & \\
\hline
\end{tabular}

PUD, peptic ulcer disease; NUD, nonulcer dyspepsia.

Table 4. Adverse Events of Eradication Medications

\begin{tabular}{lccr}
\hline \multicolumn{1}{c}{ Adverse event } & $\begin{array}{c}\text { Standard triple } \\
\text { therapy } \\
(\mathrm{n}=48)\end{array}$ & $\begin{array}{c}\text { Pronase combined } \\
\text { with standard triple } \\
\text { therapy }(\mathrm{n}=47)\end{array}$ & $\begin{array}{c}\mathrm{p}- \\
\text { value }\end{array}$ \\
\hline Bitter taste & $14(29.2)$ & $19(40.4)$ & 0.29 \\
Nausea & $4(8.3)$ & $2(4.3)$ & 0.68 \\
Diarrhea & $4(8.3)$ & $3(6.4)$ & $>0.99$ \\
Epigastric discomfort & $1(2.1)$ & $1(2.1)$ & $>0.99$ \\
Dry mouth & $1(2.1)$ & - & $>0.99$ \\
Skin rash & $1(2.1)$ & - & $>0.99$ \\
Total & $19(39.6)$ & $23(48.9)$ & 0.41 \\
Adherence $<90 \%$ & 0 & $2(4.3)$ & 0.24 \\
\hline
\end{tabular}

Data are presented as number (\%).

Among the PPIs prescribed in the eradication regimen, no single medication showed superior efficacy (lansoprazole [46] vs omperazole [25] vs pantoprazole [21] vs esomeprazole [3], $\mathrm{p}=0.45$ ).

\section{The adverse events}

A total of 48 patients (100\%) in the PAC group and 45 patients (95.7\%) in the PACE group adhered to the prescribed medications. All the patients were asked to submit self-reported questionnaire about adverse events whose rate was reported as $39.6 \%$ in the PAC group and $48.9 \%$ in the PACE group ( $\mathrm{p}=0.41$ ). The most common adverse event was bitter taste $(29.2 \%$ in PAC vs $40.4 \%$ in PACE group), followed by nausea and diarrhea. All the reported adverse events are shown in Table 4.

\section{DISCUSSION}

In this study, the overall eradication rates (ITT and PP analysis) were lower in the PACE group than in the PAC group (ITT, $56.1 \%$ vs $76.4 \%$; PP, $68.1 \%$ vs $87.5 \%$ ). These results do not correspond to the earlier randomized controlled study which reported that LAMP (lansoprazole once daily, $500 \mathrm{mg}$ of amoxicillin, $250 \mathrm{mg}$ of metronidazole and 18,000 tyrosine units of pronase thrice daily for 2 weeks) group showed significantly higher eradication rate than LAM group (ITT, 94\% vs 76.5\%; $\mathrm{p}=0.0041){ }^{7}$ Another study which used pronase 18,000 tyrosine units twice a day for 2 days showed potential benefits of pronase on the $H$. pylori eradication, even though the regimen was combined with topical anti-Helicobacter treatment, which is no longer used and the study itself was not a well-designed one to prove the efficacy of pronase. ${ }^{13}$

The first explanation for the decreased efficacy of pronase combined with standard triple therapy could be decreased gastrointestinal residence time of amoxicillin. Orally administered amoxicillin is known to be distributed in the mucous layer and surface epithelial cells of stomach. ${ }^{14}$ According to the study which evaluated the efficacy of mucoadhesive form of amoxicillin, prolonged gastrointestinal residence time of amoxicillin showed enhanced $H$. pylori clearance rate. ${ }^{15}$ However, pronase is known to disrupt and make a reduction in the thickness of SMGL, which can reduce the gastrointestinal residence time of amoxicillin. The decreased efficacy of amoxicillin in the eradication regimen could be the reason for the overall reduction of eradiation rate of pronase combined with standard triple therapy group.

The second explanation for the decreased efficacy of pronase combined with standard triple therapy could be not enough alteration of intragastric $\mathrm{pH}$. Maximal mucinolysis by pronase is known to occur at pH 6 to $8 .{ }^{4}$ Thus, intragastric neutralizer such as $\mathrm{NaHCO}_{3}$ or parasympathetic blocker such as scopolamine butylbromide have been recommended as the coadministrating agent with pronase. ${ }^{5}$ In the previous study that revealed the additive effect of pronase on the eradication of $H$. pylori, there was no coadministrating agent such as $\mathrm{NaHCO}_{3}$. Moreover, the dose of PPI was lower than in our study (lansoprazole once daily). However, extended duration was maintained (for 2 weeks) and the LAMP group achieved better eradication rate than the control group. ${ }^{7}$ Thus, the mechanism of the enhanced eradication rate of $H$. pylori is unclear and unexplainable by pronase in that study. In our study, a double dose of PPI (twice daily for 7 days) was prescribed with pronase. Moreover, NaHCO3, the coadministrating agent, was prescribed unlike to the previous study. However, all the patients were recommended to be administered the pronase and $\mathrm{NaHCO}_{3}$ at the same time with the eradication medication, which is an unusual administration method. Because patients who undergo endoscopy are generally recommended to take the pronase with $\mathrm{NaHCO}_{3} 10$ to 20 minutes before the endoscopy to allow the gastric mucus to degrade in order to enhance the visibility of the endoscopic view. However, complicated administration method decreases the compliance of eradication medication. Thus, in this trial, the administration method was simplified. 
The last explanation is an inadequate administration method of the pronase. Pronase that was used in this study is a powder form of medication which should be administered with 80 to $100 \mathrm{~mL}$ of warm water to be well dissolved and dispersed in the stomach. ${ }^{16}$ The study that evaluated the efficacy of pronase for improved visibility during endoscopy revealed that rotating the patients enhanced the visibility because of the wide dispersion. ${ }^{4}$ However, in our study, medication counseling focused only on the administration time and adverse events. More detailed medication education and counseling such as administering an adequate amount of warm water and keeping movement after the pronase administration could affect the outcome. In the randomized study that assessed the additive effect of pronase on the eradication of $H$. pylori, increased local delivery of antibiotics by disrupting SMGL was speculated for the main mechanism of increased eradication rate. ${ }^{7}$ This can be achieved by the even distribution of pronase on the gastric mucosa which is speculated to be insufficient in our study.

Another issue is the optimal dose of the pronase. According to the studies that evaluated the effectiveness of pronase for enhanced visualization of mucosa during endoscopy, 20,000 tyrosine units of pronase given 10 or 20 minutes before endoscopy achieved satisfactory visualization. ${ }^{4,16,17}$ However, in the previous randomized controlled trial that assessed the additive effect of pronase on the eradication of $H$. pylori, 18,000 tyrosine units were used. The optimal amount of pronase needed to increase the local delivery of antibiotics has not been investigated. Our study used 20,000 tyrosine units trice with $H$. pylori eradication medication. However, regarding the short duration of action time, the dose of pronase could have been insufficient.

In terms of adverse events, relatively high rates were reported as $39.6 \%$ in PAC group and $48.9 \%$ in PACE group ( $p=0.41)$ since the analysis included all the minor side effects such as bitter taste and dry mouth (Table 4$)$.

According to a study about anitimicrobial activity, pronase does not have in vitro antimicrobial activity or any synergistic effect with antibiotics against $H$. pylori. ${ }^{7}$ However, gastric secretion of amoxicillin and metronidazole, but not clarithromycin was increased by pronase in a rat model suggesting increased local delivery and transfer of antibiotics by disrupting SMGL. ${ }^{18}$

In this study, it is shown that decreased gastrointestinal residence time of amoxicillin, inadequate elevation of intragastric $\mathrm{pH}$, inappropriate administration method and dose of pronase could affect the outcome. Authors initially planned to enroll 108 patients in each treatment group to reveal the additive effect of pronase on the eradication of $H$. pylori infection. However, the interim analysis showed unexpectedly poor outcome. Thus, this trial stopped the enrollment of patients. The retrospective power analysis revealed power between $60 \%$ to $70 \%$ by Altman's normogram or Lehr's formula. ${ }^{19}$ The limitation of this interim analysis is that the poor outcome in the pronase combined with triple therapy group (PACE) is underpowered to conclude its far inferior result. However, for the welfare of the enrolled patients, this study was discontinued after a discussion among the authors.

According to this pilot trial, pronase does not have an additive effect on the eradication of $H$. pylori infection.

\section{CONFLICTS OF INTEREST}

No potential conflict of interest relevant to this article was reported.

\section{REFERENCES}

1. Graham DY, Lu H, Yamaoka Y. A report card to grade Helicobacter pylori therapy. Helicobacter 2007;12:275-278.

2. Graham DY, Shiotani A. New concepts of resistance in the treatment of Helicobacter pylori infections. Nat Clin Pract Gastroenterol Hepatol 2008;5:321-331.

3. Graham DY, Fischbach L. Helicobacter pylori treatment in the era of increasing antibiotic resistance. Gut 2010;59:1143-1153.

4. Fujii T, Iishi H, Tatsuta M, et al. Effectiveness of premedication with pronase for improving visibility during gastroendoscopy: a randomized controlled trial. Gastrointest Endosc 1998;47:382-387.

5. Koga M, Arakawa K. On the application of enzymatic mucinolysis in X-ray diagnosis of the stomach. Nihon Igaku Hoshasen Gakkai Zasshi 1964;24:1011-1031.

6. Ida K, Okuda J, Nakazawa S, et al. Clinical evaluation of premedication with KPD (pronase) in gastroendoscopy: placebocontrolled double blind study in dye scattering endoscopy. Clin Rep 1991;25:1793-1804.

7. Gotoh A, Akamatsu T, Shimizu T, et al. Additive effect of pronase on the efficacy of eradication therapy against Helicobacter pylori. Helicobacter 2002;7:183-191.

8. Hashimoto Y, Tsuiki S, Nisizawa K, Pigman W. Action of proteolytic enzymes on purified bovine submaxillary mucin. Ann N Y Acad Sci 1963;106:233-246.

9. Taniguchi Y, Yoshida Y, Kimura K, Mato M. Cytoprotection by 16,16-dimethylprostaglandin E2. Role of gastric juice and mucus gel layer. J Clin Gastroenterol 1992;14 Suppl 1:S52-S58.

10. Hazell SL, Lee A, Brady L, Hennessy W. Campylobacter pyloridis and gastritis: association with intercellular spaces and adaptation to an environment of mucus as important factors in colonization of the gastric epithelium. J Infect Dis 1986;153:658-663.

11. Shimizu T, Akamatsu T, Sugiyama A, Ota H, Katsuyama T. Helicobacter pylori and the surface mucous gel layer of the human stomach. Helicobacter 1996;1:207-218.

12. Chung JW, Lee GH, Han JH, et al. The trends of one-week firstline and second-line eradication therapy for Helicobacter pylori infection in Korea. Hepatogastroenterology 2011;58:246-250.

13. Kimura K, Ido K, Saifuku K, et al. A 1-h topical therapy for the treatment of Helicobacter pylori infection. Am J Gastroenterol 1995;90:60-63. 
14. Endo H, Yoshida H, Ohmi N, Ohta K, Higuchi S. Localization of [14C]amoxicillin in rat gastric tissue when administered with lansoprazole and clarithromycin. J Antimicrob Chemother 2001;48:923-926.

15. Patel JK, Chavda JR. Formulation and evaluation of stomachspecific amoxicillin-loaded carbopol-934P mucoadhesive microspheres for anti-Helicobacter pylori therapy. J Microencapsul 2009;26:365-376.

16. Lee GJ, Park SJ, Kim SJ, Kim HH, Park MI, Moon W. Effectiveness of premedication with pronase for visualization of the mucosa during endoscopy: a randomized, controlled trial. Clin Endosc
2012;45:161-164.

17. Chang CC, Chen SH, Lin CP, et al. Premedication with pronase or $\mathrm{N}$-acetylcysteine improves visibility during gastroendoscopy: an endoscopist-blinded, prospective, randomized study. World J Gastroenterol 2007;13:444-447.

18. Sherwood PV, Wibawa JI, Atherton JC, et al. Impact of acid secretion, gastritis, and mucus thickness on gastric transfer of antibiotics in rats. Gut 2002;51:490-495.

19. Whitley E, Ball J. Statistics review 4: sample size calculations. Crit Care 2002;6:335-341. 